\title{
Relativity of pure states entanglement
}

\author{
Karol Życzkowski ${ }^{1 *}$ and Ingemar Bengtsson ${ }^{2}$ \\ ${ }^{1}$ Centrum Fizyki Teoretycznej, Polska Akademia Nauk, \\ Al. Lotników 32/46, 02-668 Warszawa, Poland \\ ${ }^{2}$ Stockholm University, SCFAB, Fysikum \\ S106 91 Stockholm, Sweden
}

(August 6, 2001)

\begin{abstract}
Entanglement of any pure state of an $N \times N$ bi-partite quantum system may be characterized by the vector of coefficients arising by its Schmidt decomposition. We analyze various measures of entanglement derived from the generalized entropies of the vector of Schmidt coefficients. For $N \geq 3$ they generate different ordering in the set of pure states and for some states their ordering depends on the measure of entanglement used. This odd-looking property is acceptable, since these incomparable states cannot be transformed to each other with unit efficiency by any local operation. In analogy to special relativity the set of pure states equivalent under local unitaries has a causal structure so that at each point the set splits into three parts: the 'Future', the 'Past' and the set of noncomparable states.
\end{abstract}

\section{INTRODUCTION}

Due to growing interest in quantum information the properties of quantum entanglement became a subject of intensive research. Entanglement gives rise to the peculiarly quantum mechanical correlations that may exist between two subsystems. In spite of several papers published yearly on this subject we do not know the answers to even some very basic questions in this field. An example of such a question: 'Is a given quantum state (pure or mixed) of a composite system separable or entangled?' For the simplest case of $2 \times 2$ and $3 \times 2$ systems this problem is solved by the Peres-Horodeccy criterion [1,2], which offers a simple condition sufficient and necessary for separability. On the other hand, in the general case of a $K \times M$ bi-partite system such a constructive condition remains still unknown [3., 4 .

Another important issue is the question: 'How much is a given quantum state entangled?'. In other words we are looking for a function of the states that quantifies entanglement. Since we do not quite understand what entanglement means this is a difficult question, but what we can do is to write down conditions that such a measure has to satisfy. A key condition is that the expected entanglement cannot increase unless the two subsystems interact in a quantum mechanical fashion. We therefore require monotonicity under local operations and classical communication (LOCC) [5] [6]. The idea is that we have two subsystems A and B and two experimenters Alice and Bob who are allowed to manipulate one subsystem each in any way they please. They are also allowed to tell each other, using classical communication, what unitary transformations and measurements they perform on their respective subsystems so that Alice can allow her manipulations to depend on what Bob does and what the results of his measurements are (and conversely for Bob). There exists a very beautiful characterization of LOCC operations due to Nielsen [7] which we will quote in due course.

Now it is known [8] that monotonicity under LOCC operations does not suffice to single out a unique measure of entanglement. A major point of the present paper is precisely to study this non-uniqueness in detail. The situation changes if we consider the asymptotic regime, in which it is assumed that we have a source producing infinitely many copies of our quantum system. In this situation suitable continuity requirements can be added to the list of properties that an entanglement measure should satisfy, and there are uniqueness theorems [9] that show that on pure states the only measure that fullfills all the axioms must agree with a particular measure known as the entropy of entanglement when evaluated on pure states. In the present paper we stay in the regime of one copy however.

Classifying measures of entanglement and selecting the physically relevant ones is a subject of vivid recent interest [8.9 14, 15]. One line of research starts with the investigation of invariants of local unitary transformations, like Schmidt coefficients for pure states [16]. Several such invariants for mixed states of a bi-partite system were recently found 17 23], and one may try to construct existing measures of entanglement out of them or define new measures with attractive properties. Another possible approach is to quantify the entanglement by the distance of the given state to the closest separable state [10:24], or to look for the best separable approximation of an entangled state [25].

*on leave from Instytut Fizyki, Uniwersytet Jagielloński, ul. Reymonta 4, 30-059 Kraków, Poland 
The aim of this paper is to elucidate some geometrical properties of the entanglement of pure states of a composite $N \times N$ system. Using the concept of the Schmidt decomposition we illustrate the observation [8] that in the regime of one copy there exists infinitely many measures of entanglement which generate different orderings of pure states. We establish a link between different measures based on the generalized entropies of the vector of Schmidt coefficients and the various distances between closest separable states [10].

Applying Nielsen's characterization of LOCC, that is using the concept of majorization [7,26] (to be introduced below), we show that any state $|\psi\rangle$ gives rise to a natural decomposition of the set of all pure states into four sets: a) the measure zero set of interconvertible states which may be obtained from $|\psi\rangle$ by local unitary transformation, b) the states accessible from $|\psi\rangle$ by nonunitary local transformations, c) the states which can be transformed locally into $|\psi\rangle$, and d) - the set of incomparable states. Once all pure states that can be transformed into each other by local unitary transformations have been identified with each other, the decomposition consists of only three sets, b), c) and d). This picture mimics the well known structure of the light cone in special relativity, which divides the space-time into 'Future', 'Past' and the set of space-like, incomparable events. Hence our title (see also [27]). The same analogy works for the entirely different problem of non-unitary evolution of mixed states under the action of external random fields. Interestingly, in this case the direction of the arrow of time is reversed.

The paper is organized as follows. In section II we review the definitions of the Schmidt decomposition of a pure state, quantum Rényi entropies and majorization, while in Section III we discuss different measures of entanglement, analyze nonhermitian evolution of the density matrices, for which the majorization theory is applicable, and the corresponding local transformations of pure states. Propositions emphasizing the geometrical interpretation of the Weyl chamber consisting of ordered eigenvalues of a density matrix (or ordered vector of the Schmidt coefficients for a pure state of a bi-partite system) are proved in Appendix A.

\section{MATHEMATICAL TOOLS AND DEFINITIONS}

\section{A. Schmidt decomposition}

Consider a pure state $|\psi\rangle$ of a composite Hilbert space $\mathcal{H}=\mathcal{H}_{A} \otimes \mathcal{H}_{B}$ of size $N^{2}$. Introducing an orthonormal basis $\{|n\rangle\}_{n=1}^{N}$ in each subsystem, we may represent the state as

$$
|\psi\rangle=\sum_{n=1}^{N} \sum_{m=1}^{N} C_{m n}|n\rangle \otimes|m\rangle .
$$

The complex matrix of coefficients $C$ of size $N$ needs not to be Hermitian nor normal. Its singular values (i.e. square roots of eigenvalues $\lambda_{k}$ of the positive matrix $C^{\dagger} C$ ) determine the Schmidt decomposition [28, 16, 29]

$$
|\psi\rangle=\sum_{k=1}^{N} \sqrt{\lambda_{k}}|k \prime\rangle \otimes\left|k^{\prime \prime}\right\rangle,
$$

where the basis in $\mathcal{H}$ is transformed by a local unitary transformation $U \otimes V$. Thus $|k \prime\rangle=U|k\rangle$, and $|k \prime \prime\rangle=V|k\rangle$, where $U$ and $V$ are the matrices of eigenvectors of $C^{\dagger} C$ and $C C^{\dagger}$, respectively. The remarkable thing is that only a single sum is involved. In the generic case of a non-degenerate vector $\vec{\lambda}$, the Schmidt decomposition is unique up to two unitary diagonal matrices, up to which the matrices of eigenvectors $U$ and $V$ are determined. The normalization condition $\langle\psi \mid \psi\rangle=1$ enforces $\sum_{k=1}^{N} \lambda_{k}=1$. Thus the vector $\vec{\Lambda}=\left(\lambda_{1}, \ldots, \lambda_{N}\right)$ lives in the $(N-1)$ dimensional simplex $\mathcal{S}_{N}$. Note that the Schmidt coefficients $\lambda_{k}$ do not depend on the initial basis $|n\rangle \otimes|m\rangle$, in which the analyzed state $|\psi\rangle$ is represented. They are invariant with respect to any local operations $U_{A} \otimes U_{B}$, and thus they may serve as ingredients of any measure of entanglement. The Schmidt simplex is almost but not quite the same as the space of orbits under local unitary transformations; since we can change the ordering of the eigenvalues by means of local unitaries some further identification of points in the Schmidt simplex has to be done before we have the space of orbits. This is discussed below (section IIID).

The Schmidt coefficients of a pure state $|\psi\rangle$ are equal to the eigenvalues of the reduced density operator, obtained by partial tracing, $\rho^{A}=\operatorname{tr}_{B}(|\psi\rangle\langle\psi|)$. The pure state is called entangled if it can not be represented in the product form $|\psi\rangle=\left|\psi_{A}\right\rangle \otimes\left|\psi_{B}\right\rangle$, where $\left|\psi_{A}\right\rangle \in \mathcal{H}_{A}$ and $\left|\psi_{B}\right\rangle \in \mathcal{H}_{B}$. This is the case if and only if there exists only one non-zero Schmidt coefficient, $\lambda_{1}=1$. 


\section{B. Entangled mixed states}

Mixed states $\rho=\sum_{i} p_{i}\left|\varphi_{i}\right\rangle\left\langle\varphi_{i}\right|$ with $p_{i}>0$ and $\sum_{i} p_{i}=1$ will also be useful in our considerations. A state $\rho$ is called a product state if it can be represented as a tensor product, $\rho_{\text {prod }}=\rho^{A} \otimes \rho^{B}$, where $\rho^{A}$ acts in $\mathcal{H}_{A}$ and $\rho^{B}$ acts in $\mathcal{H}_{B}$. A mixed state $\rho$ is called separable if it can be represented as a convex combination of product states, $\rho_{\text {sep }}=\sum_{j} q_{j} \rho_{j}^{A} \otimes \rho_{j}^{B}$, where $q_{j}>0$ and $\sum_{j} q_{j}=1$. A mixed state which is not separable is called entangled. It is easy to see that for pure states both definitions are consistent.

\section{Measures of entanglement}

There exist several different possibilities to quantify quantum entanglement - for a review on this subject see e.g. [4, 15]. Following Vedral and Plenio [10] we assume that each measure of entanglement $E(\rho)$ has to satisfy the following conditions:

(E1) $E(\rho)=0$ if $\rho$ is separable, (the condition 'if and only if' occurs to be too strong [15])

(E2) Local unitary operations do not change the entanglement, i.e. $E(\rho)=E\left(U_{A} \otimes U_{B} \rho U_{A}^{\dagger} \otimes U_{B}^{\dagger}\right)$

(E3) The expected entanglement cannot increase under operations involving local measurements and classical communication (LOCC), followed by post-selection:

$$
\sum_{i=1}^{M} \operatorname{tr} \rho_{i} E\left(\frac{\rho_{i}}{\operatorname{tr} \rho_{i}}\right) \leq E(\rho),
$$

where $\rho_{i}=V_{i} \rho V_{i}^{\dagger}$, each of the operators $V_{i}$ is local, i.e. $V_{i}=V_{i}^{A} \otimes V_{i}^{B}$, and the set of $M$ these operators defines an positive operator valued measure (POVM) [16], i.e. $\sum_{i=1}^{M} V_{i}^{\dagger} V_{i}=\mathbb{I}$. Note that the definition allows the actions on the two subsystems to be correlated; this is how classical communication enters. Note also that it is the expected entanglement that cannot increase - if we end up with a statistical ensemble of states there may be a non-zero probability of increased entanglement.

Sometimes one imposes further requirements

(E4) Additivity: $E\left(\rho_{1} \otimes \rho_{2}\right)=E\left(\rho_{1}\right)+E\left(\rho_{2}\right)$,

(E5) Continuity: $E$ is a continuous function of $\rho$,

or

(E5') Asymptotic continuity: $E$ is a continuous function of the fidelity for $n$ copies of the same pure state, $|\psi\rangle^{\otimes}$ in the asymptotic limit $n \rightarrow \infty$,

the necessity of which is still disputed in the literature [8,15,9]. Condition (E4) is most welcome but difficult to prove in the general case of arbitrary density matrices, while condition (E5) is satisfied by many different measures. On the other hand, condition (E5') is rather strong: assuming that the measure of entanglement fulfills some technical conditions which quantify asymptotic continuity one may show 8. 15] that for pure states it is proportional to the entropy of entanglement, $E(|\psi\rangle\langle\psi|)=H_{N}(|\psi\rangle)=-\operatorname{tr}\left(\rho^{A}\right) \ln \left(\rho^{A}\right)$. Another set of axioms for the measure of entanglement which singles out the entropy of entanglement is given by Rudolph [41]. Such requirements are indeed appropriate in the asymptotic regime but it remains interesting to investigate the regime of one copy where only the first three conditions are imposed. One of the aims of this paper is to emphasize that there exist several reasonable measures of entanglement, which for pure states are different from the entropy of entanglement.

\section{Quantum Rényi entropies}

Consider an $N$ dimensional vector $\vec{x}$ with non-negative components normalized as $\sum_{i=1}^{N} x_{1}=1$. The distribution of $x_{i}$ may be described by the Shannon (information) entropy $H_{N}:=-\sum_{i=1}^{N} x_{i} \ln x_{i}$. A more general family of quantities characterizing the components of $\vec{x}$ is provided by the Rényi entropies [31] often used in information theory [32];

$$
H_{\alpha}(\vec{x}):=\frac{1}{1-\alpha} \ln \left[\sum_{i=1}^{N} x_{i}^{\alpha}\right] .
$$


As usual, in the definition of entropies we adopt the convention that $0 \ln 0=0$, if necessary. The non-negative number $\alpha \neq 1$ is a free parameter labeling the generalized entropy. It is easy to see that $\lim _{\alpha \rightarrow 1} H_{\alpha}=H_{N}$, so for consistency we will write sometimes $H_{1}$ for $H_{N}$. The quantities $H_{\alpha}(\vec{x})$ vary from zero (for $\vec{x}$ with one non-zero component) to $\ln N$, for the vector $\vec{x}_{*}$ with all components equal, $x_{i}=1 / N$. It is possible to show that the generalized entropy is a non-increasing function of its parameter: for any $\vec{x}$ and $\alpha_{2}>\alpha_{1}$, the inequality $H_{\alpha_{2}}(\vec{x}) \leq H_{\alpha_{1}}(\vec{x})$ holds [32].

Some special cases of $H_{\alpha}$ are of particular interest. Let $r$ denote the number of non-zero components of the vector $\vec{x}, x_{1}$ be its largest component, and $|\vec{x}|=\left(\sum_{i=1}^{r} x_{i}^{2}\right)^{1 / 2}$ be its length. Then

$$
H_{0}(\vec{x})=\ln r, \quad H_{2}(\vec{x})=-\ln |\vec{x}|^{2}, \quad H_{\infty}(\vec{x})=-\ln x_{1} .
$$
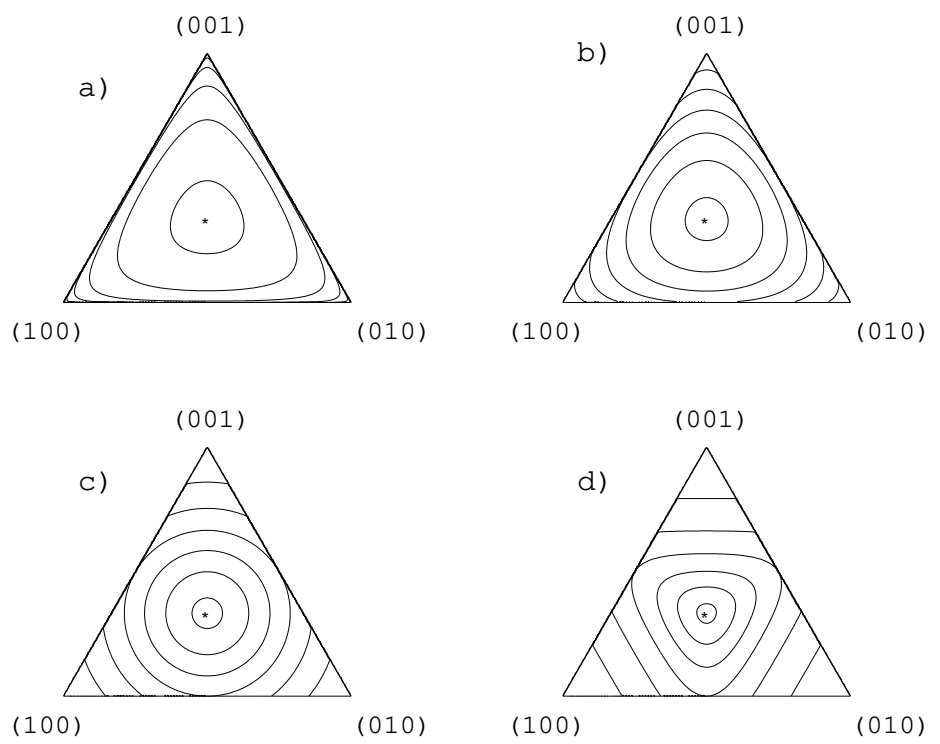

FIG. 1. Iso-entropy curves $H_{\alpha}(\vec{x})$ in the space of $N=3$ vectors $\vec{x}$ plotted for (a) $\alpha=1 / 3$, (b) $\alpha=1$ (also contour lines of the Shannon entropy), (c) $\alpha=2$ (circles of points equidistant from $\vec{x}_{*}$ ), and (d) $\alpha=5$.

To get some experience with the properties of generalized entropies let us look at Fig. 1, which shows the iso-entropy curves for $N=3$. The equilateral triangle, of side length $\sqrt{2}$, lies in the plane $x_{3}=1-x_{2}-x_{1}$ and its corners are labeled by the co-ordinates $\left(x_{1}, x_{2}, x_{3}\right)$. At these three points the generalized entropies attain their minima, $H_{\alpha}=0$, for any values of $\alpha \geq 0$. The maximum $H_{\alpha}=\ln 3$ is achieved at the center of the triangle, which represents the uniform vector $\vec{x}_{*}$. The maximum is rather flat for $\alpha=1 / 3$, as shown in Fig. 1 1a. For even smaller, non-negative values of $\alpha$ the isoentropy lines become parallel to the closest side of the triangle. The entropy $H_{0}$ vanish at the corners of the triangle, is equal to $\ln 2$ at its sides and equals to $\ln 3$ for any point inside the triangle. The other example, $\alpha=5$, presented in Fig. 1 $1 \mathrm{~d}$. is similar to the limiting case $H_{\infty}$, for which the iso-entropy curves are parallel to the most distant side of the triangle.

For any two values of $\alpha$ the isoentropy lines intersect. Thus any pair of two different generalized entropies induces a different ordering of vectors $\vec{x}$. In our considerations the vector $\vec{x}$ will represent two entirely different objects:

a) the spectrum $\vec{d}$ of a $N=3$ density matrix $\rho$

or

b) the vector of the Schmidt coefficients $\vec{\Lambda}$ of a pure state of the $3 \times 3$ composite system.

Thus the pictures of isoentropy lines may have two entirely different meanings. In the former case a), the entropy $H_{N}$ is equivalent to the von Neumann entropy, $S_{N}(\rho)=-\operatorname{Tr}[\rho \ln \rho]$, while other quantum Renyi entropies

$$
S_{\alpha}(\rho)=\frac{1}{1-\alpha} \ln \operatorname{tr} \rho^{\alpha}
$$

play the role of different measures of degree of mixing [33, 34]. The entropy $S_{0}$ is a function of the rank $r$ of the density matrix, i.e. the number of positive eigenvalues of $\rho$. Thus it is not a continuous function of its argument in contrast to generalized entropies $S_{\alpha}$ with $\alpha>0$. For $\alpha=2$ we have $S_{2}=-\ln \left[\operatorname{Tr} \rho^{2}\right]=\ln R$, where the purity (also called inverse participation ratio) $R(\rho)=\left[\operatorname{Tr} \rho^{2}\right]^{-1}$ describes the "effective number of states" involved in the mixture $\rho$ and varies 
from unity (for pure states) to $N$ (for $\rho_{*}=\mathbb{I} / 3$ ). The purity of any state depends only on its Hilbert-Schmidt distance from the maximally mixed state $\rho_{*}$, represented by the center of the triangle. Thus the curves of constant $R$ have the shape of a circle, and the corners - the points most distant form $\rho_{*}$ - denote three orthogonal pure states. Note that due to the presence of the logarithm in the definition (2.6) the generalized entropies are additive for product states,

$$
S_{\alpha}\left(\rho_{1} \otimes \rho_{2}\right)=S_{\alpha}\left(\rho_{1}\right)+S_{\alpha}\left(\rho_{2}\right)
$$

for any $\alpha \geq 0$.

In the latter case b) the centers of the triangles plotted in Fig. 1 represent the maximally entangled states $\left|\psi_{*}\right\rangle=$ $(|11\rangle+|22\rangle+|33\rangle) / \sqrt{3}$ and the corners denote separable states. The generalized entropy of a vector of the Schmidt coefficients equals the Renyi entropy of the reduced density matrices, $H_{\alpha}(|\psi\rangle)=S_{\alpha}\left[\operatorname{tr}_{B}(|\psi\rangle\langle\psi|)\right]=S_{\alpha}\left[\operatorname{tr}_{A}(|\psi\rangle\langle\psi|)\right]$. In particular $H_{N}$ coincides with the entropy of entanglement of the corresponding pure states of the composite $3 \times 3$ system. As discussed later, all generalized entropies $H_{\alpha}$ for $\alpha \geq 0$ fulfill the conditions (E1)-(E3), and may thus serve as legitimate measures of the pure state entanglement. Moreover, for any product pure states these measures are additive, $H_{\alpha}\left(\left|\psi_{1}\right\rangle \otimes\left|\psi_{2}\right\rangle\right)=H_{\alpha}\left(\left|\psi_{1}\right\rangle\right)+H_{\alpha}\left(\left|\psi_{2}\right\rangle\right)$. This property, following from (2.7), was pointed out by Vidal [8].

Note that an analogous construction of isoentropy hypersurfaces in the $(N-1)$ dimensional simplex may represent mixed states acting in $\mathcal{H}_{N}$ or pure states of the composite $N \times N$ system. For any $N \geq 3$ the entropies $H_{\alpha}(\vec{x})$ provide not equivalent measures of uniformity/disorder in the distribution of the components $x_{i}$.

\section{E. Majorization}

The question, which of any two vectors is more 'mixed' (or more disordered) can be approached by the theory of majorization [35, which allows one to introduce a partial order into the this set. Consider two vectors $\vec{x}$ and $\vec{y}$, consisting of $N$ non-negative components each. We order the components of both vectors in a decreasing order, (what is sometimes emphasized by the notation $x^{\downarrow}$ ), and assume that they are normalized in the sense $\sum_{i=1}^{N} x_{i}=\sum_{i=1}^{N} y_{i}=1$. We say that $\vec{x}$ is majorized by $\vec{y}$, written $\vec{x} \prec \vec{y}$, if

$$
\sum_{i=1}^{k} x_{i} \leq \sum_{i=1}^{k} y_{i}
$$

for $k=1,2, \ldots, N-1$. Since the sum of all components of both vectors are equal, we obtain an equivalent formulation of majorization

$$
\sum_{i=l}^{N} x_{i} \geq \sum_{i=l}^{N} y_{i}
$$

for all $l=2, \ldots, N$. Vaguely speaking, the vector $\vec{x}$ is more 'mixed' than the vector, $\vec{y}$ and its distribution function grows slower with the index $i$ (see Fig. 2).
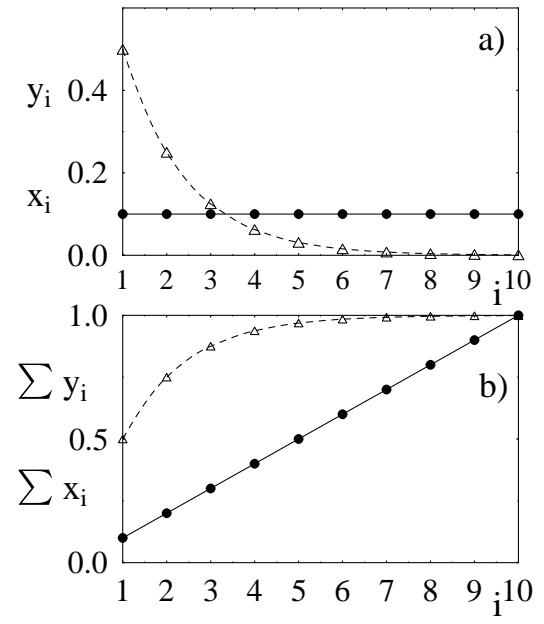

FIG. 2. Idea of majorization: (a) the ordered vector $x^{\downarrow}=\left\{x_{1}, \ldots, x_{10}\right\}(\bullet)$ is majorized by $\vec{y}=\left\{y_{1}, \ldots, y_{10}\right\}(\triangle)$, since the corresponding distribution function takes larger values (b), and both functions do not cross. 
The functions which preserve the majorization order are called Schur convex,

$$
\vec{x} \prec \vec{y} \text { implies } f(\vec{x}) \leq f(\vec{y}) .
$$

Examples of Schur convex functions include $f_{q}(\vec{x})=\sum_{i=1}^{N} x_{i}^{q}$ for any $q \geq 1, \tilde{f}_{q}(\vec{x})=-\sum_{i=1}^{N} x_{i}^{q}$ for any $0<q<1$, and $f_{e}(\vec{x})=\sum_{i=1}^{N} x_{i} \ln x_{i}$ [35], while these functions with reversed sign are Schur concave. To show a simple application of the theory of majorization let us then consider two density matrices $\rho_{x}$ and $\rho_{y}$, with spectra $\vec{x}$ and $\vec{y}$, respectively. If $\vec{x} \prec \vec{y}$ then due to Schur-convexity $H_{\alpha}(\vec{x}) \geq H_{\alpha}(\vec{y})$ for $\alpha \geq 0$, since at $\alpha=1$ the prefactor $1 /(1-\alpha)$ in (2.4) changes the sign and the direction of the inequality is reversed.

A classical theorem by Hardy, Littlewood and Polya states that $\vec{x} \prec \vec{y}$ if and only if there exists a bistochastic matrix $B$ such that $\vec{x}=B \vec{y}$. A matrix $B_{i j}$ with non-negative elements is called bistochastic if sum of all elements in each row and each column equals to unity. As shown later by Horn [36] this theorem can be strengthened by replacing the word bistochastic by unistochastic. A bistochastic matrix $B$ is called unistochastic, if there exists an unitary matrix $U$ such that $B_{i j}=\left|U_{i j}\right|^{2}$. All $N=2$ bistochastic matrices are unistochastic, but this is not true for $N \geq 3$ [35].

Another useful feature of majorization is related to the so called $T$-transform. It is a matrix of size $N$ which acts as identity on all but two dimensions where it has the form of a bistochastic matrix

$$
\tilde{T}=\left[\begin{array}{cc}
t & 1-t \\
1-t & t
\end{array}\right]
$$

so $t \in[0,1]$. One can prove [35, 37] that $\vec{x} \prec \vec{y}$ if and only if there exist a set of $T$-transforms $T_{1}, T_{2}, \ldots, T_{k}$ that $x=T_{1} T_{2} \cdots T_{k} y$. The maximal number of the $T$-transforms needed is not larger than $N-1$. A simple proof of this fact and of the Horn lemma is recently provided by Nielsen [38].

Sometimes it is useful to compare non normalized vectors, such that $\sum_{i=1}^{N} x_{i} \neq \sum_{i=1}^{N} y_{i}=1$. In such a case one introduces the relation of weak majorization [35] defining $\vec{x} \prec{ }_{w} \vec{y}$ if

$$
\sum_{i=1}^{k} x_{i} \leq \sum_{i=1}^{k} y_{i} \quad \text { for } \quad k=1,2, \ldots, N .
$$

Then vector $\vec{x}$ is said to be weakly submajorized by $\vec{y}$, or alternatively, weakly majorized from below.

Majorization technique allows one to obtain effective necessary criteria for separability of mixed states, as recently shown by Nielsen and Kempe [39]: If a mixed state $\rho$ is separable then $\vec{d} \prec \vec{d}_{A}$ and $\vec{d} \prec \vec{d}_{B}$ where $\vec{d}_{A}$ and $\vec{d}_{B}$ denote the spectra of the reduced operators $\rho^{A}$ and $\rho^{B}$. Since the Rényi entropies are Schur concave, one obtains

$$
S_{\alpha}\left(\rho^{A}\right) \leq S_{\alpha}(\rho) \quad \text { and } \quad S_{\alpha}\left(\rho^{B}\right) \leq S_{\alpha}(\rho)
$$

for any separable $\rho$ and $\alpha \geq 0$, which generalizes earlier results of Horodeccy [34].

\section{PURE STATES ENTANGLEMENT}

\section{A. Distance to the closest separable state}

For any entangled state $\rho_{\text {ent }}$ the distance to the closest separable state $\rho_{c}$ may be considered as a measure of entanglement. Each metric in the space of mixed quantum states will thus define a certain measure of entanglement. Vedral and Plenio have shown [10] that the Bures distance [40], $D_{B}\left(\rho_{1}, \rho_{2}\right)^{2}=2-2 \operatorname{tr}\left|\rho_{1}^{1 / 2} \rho_{2} \rho_{1}^{1 / 2}\right|$, to the closest separable state fulfills the required conditions $(E 1)-(E 3)$. For a pure state written in the Schmidt decomposition as in (2.2) the closest separable state is

$$
\rho_{c}=\sum_{k=1}^{N} \lambda_{k}|k k\rangle\langle k k|,
$$

(this result is proved in 10] for $N=2$ and stated for larger $N$ ). The state $\rho_{c}$ is typically mixed and its squared Bures distance to the pure state $\rho_{\psi}=|\psi\rangle\langle\psi|$ reads

$$
D_{B}^{2}\left(\rho_{\psi}, \rho_{c}\right)=2-2 \sqrt{\sum_{k=1}^{N} \lambda_{k}^{2}}=2-2 \sqrt{1 / R} .
$$


The inverse participation ratio $R$ is closely related with the generalized entropy of order 2 , namely $R=\exp \left(H_{2}\right)$. Therefore the circles around the maximally entangled state, forming the isoentropy lines for $H_{2}$ (see Fig. 1c), form also the lines of the same Bures entanglement. The same concerns generalized concurrence, another measure of entanglement recently introduced by Rungta et al. [41.

Vedral and Plenio discussed also the quantum relative entropy, $S\left(\rho_{1} \mid \rho_{2}\right):=\operatorname{tr}\left[\rho_{1}\left(\ln \rho_{1}-\ln \rho_{2}\right)\right]$. Although this quantity does not induce a true metric (e.g. it is not symmetric), the entropy $S\left(\rho_{\text {ent }} \mid \rho_{c}\right)$ satisfies $(E 1)-(E 3)$ and may be considered as a measure of entanglement. Moreover, they showed that the state (3.1) is also the 'closest' separable state to the pure state $\rho_{\psi}$ and the smallest relative entropy $S_{\min }=\sum_{k=1}^{N} \lambda_{k} \ln \lambda_{k}$ coincides with the Shannon entropy $H_{N}$ of the vector of the Schmidt coefficients. This quantity, often briefly called entanglement of the pure state $\rho_{\psi}$, is given a special physical meaning, since the probability of not distinguishing between the analyzed entangled state $\rho_{\text {ent }}$ and the closest separable state $\rho_{c}$ after $n$ measurements behaves as $\exp \left[-n S\left(\rho_{\text {ent }} \mid \rho_{c}\right)\right]$ [10]. Furthermore, the entanglement of formation [6.42] of any mixed state $\rho$, defined as the minimal average entanglement of pure states, the mixture of which generates $\rho$, for a pure state reduces to the entropy of entanglement $H_{N}$.

For any entangled pure state (2.2) one may also look for the closest separable pure state. This problem was recently considered by Lockhart and Steiner [43], who show that the state $|\phi\rangle:=\left|1^{\prime} 1^{\prime \prime}\right\rangle\left\langle 1^{\prime} 1^{\prime \prime}\right|$ corresponding to the largest Schmidt coefficient $\lambda_{1}:=\max \left\{\lambda_{i}\right\}$ is the closest. They were using the Hilbert-Schmidt distance, $D_{H S}\left(\rho_{1}, \rho_{2}\right)=\left[\operatorname{tr}\left(\rho_{1}-\rho_{2}\right)^{2}\right]^{1 / 2}$, and found $D_{H S}\left(\rho_{\psi}, \rho_{\phi}\right)=\left[2-2 \lambda_{1}\right]^{1 / 2}$. However, the projective cross-ratio $\kappa:=|\langle\psi \mid \phi\rangle|^{2}=\lambda_{1}$ is the only parameter determining the standard distances in the space of pure state, e.g. the Bures distance $D_{B}\left(\rho_{\psi}, \rho_{\phi}\right)=[2(1-\sqrt{\kappa})]^{1 / 2}$, the trace distance $D_{t r}\left(\rho_{\psi}, \rho_{\phi}\right)=2[1-\kappa]^{1 / 2}$, and the Fubini-Study distance $D_{F S}(|\psi\rangle,|\phi\rangle)=\frac{1}{2} \arccos (2 \kappa-1)=\arccos (\sqrt{\kappa})$. All these functions are monotone, so the measure of entanglement defined as the distance (any of the above) to the closest separable pure state defines an ordering of the pure states identical with that given by the Chebyshev-like like entropy $H_{\infty}=-\ln \lambda_{1}$. We have thus shown that three different settings of the problem of finding for any pure state of an $N \times N$ system the closest separable state reduce to the generalized entropies with $\alpha=1,2$ and $\infty$.

On the other hand one should not expect that every reasonable measure of pure states entanglement satisfying conditions (E1)-(E3) is neccessarily a function of one of the entropies (2.4). As a counterexample let us mention the coefficients of the characteristic polynomial of the nontrivial block of the Gram matrix introduced in [23]. They can be expressed as symmetric functions of all Schmidt coefficients, e.g. $\sum_{i>j} \lambda_{i} \lambda_{j}, \sum_{i>j>l} \lambda_{i} \lambda_{j} \lambda_{l}, \ldots$, where the summation goes over all possible sets of $k$ indices, $k=2, \ldots, N$ [44]. All these functions are Schur-concave [35], and thus entanglement monotones, although in the general case, (for $N \geq 3$ ) they are not functions of the generalized entropies.

\section{B. $2 \times 2$ system - only one ordering of pure states}

For pedagogical reasons we start to analyze the pure state entanglement with the simplest $2 \times 2$ system. Pure states of this $N=4$ system may be parametrized as

$$
\left|\psi_{4}\right\rangle=\left(\cos \vartheta_{3}, \sin \vartheta_{3} \cos \vartheta_{2} e^{i \varphi_{3}}, \sin \vartheta_{3} \sin \vartheta_{2} \cos \vartheta_{1} e^{i \varphi_{2}}, \sin \vartheta_{3} \sin \vartheta_{2} \sin \vartheta_{1} e^{i \varphi_{1}}\right),
$$

where $\vartheta_{k} \in[0, \pi / 2]$, and $\varphi_{k} \in[0,2 \pi)$ for $k=1,2,3$. The states $|\psi\rangle$ belong to the complex projective manifold $\mathbb{C} P^{3}$. This space is compact and has 6 real dimensions, e.g. three polar angles $\vartheta_{i}$ and three azimuthal angles $\varphi_{i}$.

To get a better understanding of $\mathbb{C} P^{3}$, let us recall the structure of the space of pure states for systems of even lower dimension. The set of the $N=2$ pure states, $\left|\psi_{2}\right\rangle=\left(\cos \vartheta, \sin \vartheta e^{i \phi}\right)$, is described by the Bloch sphere $\mathbb{C} P^{1} \sim S^{2}$. The sphere may be drawn in a simplified way by an interval (a meridian labeled by $\vartheta \in[0, \pi]$ ), each point of which represents a circle (a parallel $\vartheta=$ const with $\varphi \in[0,2 \pi)$ ). At both poles the circles reduce to a point - see Fig.2.

The 4-dimensional manifold $\mathbb{C} P^{2}$ of the $N=3$ pure states can be parametrized as $\left|\psi_{3}\right\rangle=$ $\left(\cos \vartheta_{2}, \sin \vartheta_{2} \cos \vartheta_{1} e^{i \varphi_{2}}, \sin \vartheta_{2} \sin \vartheta_{1} e^{i \varphi_{1}}\right)$ where $\vartheta_{1} \in[0, \pi / 2], \vartheta_{2} \in[0, \pi / 2)$ and $\varphi_{1}, \varphi_{2} \in[0,2 \pi)$. These local coordinates allow us to describe (almost all of) this space as shown in Fig.2b. The angles $\left(\vartheta_{1}, \vartheta_{2}\right)$, describe a point in the positive octant of a sphere $S^{2}$ (or in an equilateral triangle, what is topologically equivalent) which represents entire 2 -torus formed of both phases $\varphi_{i}$ [45]. Each point on one the three edges of the octant represents a circle, so each entire edge corresponds to a sphere. Note that three corners of the triangle are not the 'corners' in $\mathbb{C} P^{2}-$ in the same sense as for $N=2$ the poles (the edges of the meridian) are topologically equivalent to all other points on the sphere. 


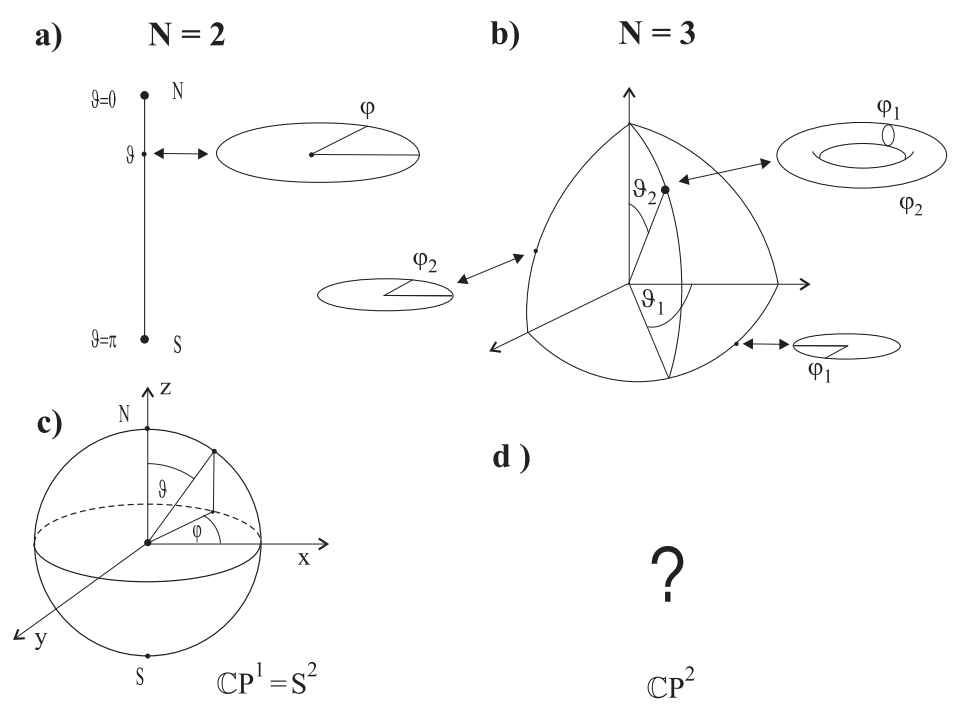

FIG. 3. The Bloch sphere, $S^{2}=\mathbb{C} P^{1}$, of all pure states of a two levels system (a) may be drawn schematically as a line, each point of which represents a circle (c). In the same, simplified manner we depict $\mathbb{C} P^{2}$ - the set of all pure states of the $N=3$ system (b). Each point inside the octant of a two-sphere, associated with the angles $\left(\vartheta_{1}, \vartheta_{2}\right)$, represents a torus $T^{2}$ spanned by the phases $\left(\varphi_{1}, \varphi_{2}\right)$. Each point at the edges of the octant denotes a circle, so each of three edges represents a sphere. The questionmark represents the 4 dimensional manifold $\mathbb{C} P^{2}$ that we are not able to reproduce exactly in the picture.

These two examples help us to imagine the structure of the space $\mathbb{C} P^{3}$ of the $N=4$ pure states. It may thus be represented as a $1 / 16$ part of the hypersphere $S^{3}$, parametrized by the angles $\vartheta_{k}$. Each point inside such a 'hyperoctant' (or simpler, the tetrahedron) represents a 3-torus spanned by the phases $\varphi_{k}$, each point on the face of the simplex a 2-torus and each point of the edge a circle. In general all points of $\mathbb{C} P^{3}$ are equivalent. This symmetry becomes broken if out of these $N=4$ system we distinguish two two-levels subsystems.

The corners of the tetrahedron represent mutually orthogonal states; let us use the standard notation and label them $(--),(-+),(+-)$, and $(++)$. In principle one could find analytically the entropy of entanglement $H_{1}$ (or any other generalized entropy $H_{\alpha}$ ) for each point of the simplex (and each choice of the phases $\varphi_{k}$ ). To get a more transparent picture we prefer to plot $H_{1}(|\psi\rangle)$ at the surface of the tetrahedron - see Fig.4.

Four corners represent the separable states. The same concerns four edges of the simplex irrespective of the phases running along the circles. Find two maximally entangled Bell states proportional to $|+-\rangle+|-+\rangle$ and $|++\rangle+|--\rangle$ localized in the middle of two non-connected edges. The other two orthogonal entangled states, $(|+-\rangle+|-+\rangle) / \sqrt{2}$ and $(|++\rangle-|--\rangle) / \sqrt{2}$, contain non-zero phase, (minus sign) and do not belong to this plot. The structure of the picture changes with other choices of the basis. If one uses the Bell basis of the four maximally entangled states and places them into four corners then the separability of the edge states depends on the phases $\varphi$.

One may portray any other generalized entropy $H_{\alpha}(|\psi\rangle)$ in a similar plot. However, in this case of pure states of the $2 \times 2$ system, all measures of the entanglement are equivalent in the sense that one measure is a function of the other one. Therefore any two entanglement measures, $E_{1}$ and $E_{2}$, generate the same ordering [46]

$$
E_{1}\left(\rho_{1}\right)>E_{1}\left(\rho_{2}\right) \Leftrightarrow E_{2}\left(\rho_{1}\right)>E_{2}\left(\rho_{2}\right)
$$

for any density operators representing pure states, $\rho_{i}=\left|\psi_{i}\right\rangle\left\langle\psi_{i}\right|$. This is due to the fact that for $N=2$ the entanglement of any pure state is completely characterized by only one relevant parameter - the Schmidt angle $\beta \in[0, \pi / 4]$, such that $\lambda_{1}=\cos ^{2}(\beta)$ while $\lambda_{2}=\sin ^{2}(\beta)$. The distribution of the Schmidt angle for random pure states distributed according to the natural, unitarily invariant measure on $\mathbb{C} P^{3}$ is given by $P(\beta)=3 \cos (2 \beta) \sin (4 \beta)$. A simple integration allows us to find the mean angle, $\langle\beta\rangle_{\mathbb{C} P^{3}}=1 / 3$ which incidentally equals the mean entropy of entanglement, $\left\langle H_{1}(|\psi\rangle)\right\rangle_{\mathbb{C} P^{3}}=1 / 3[47,48]$. 


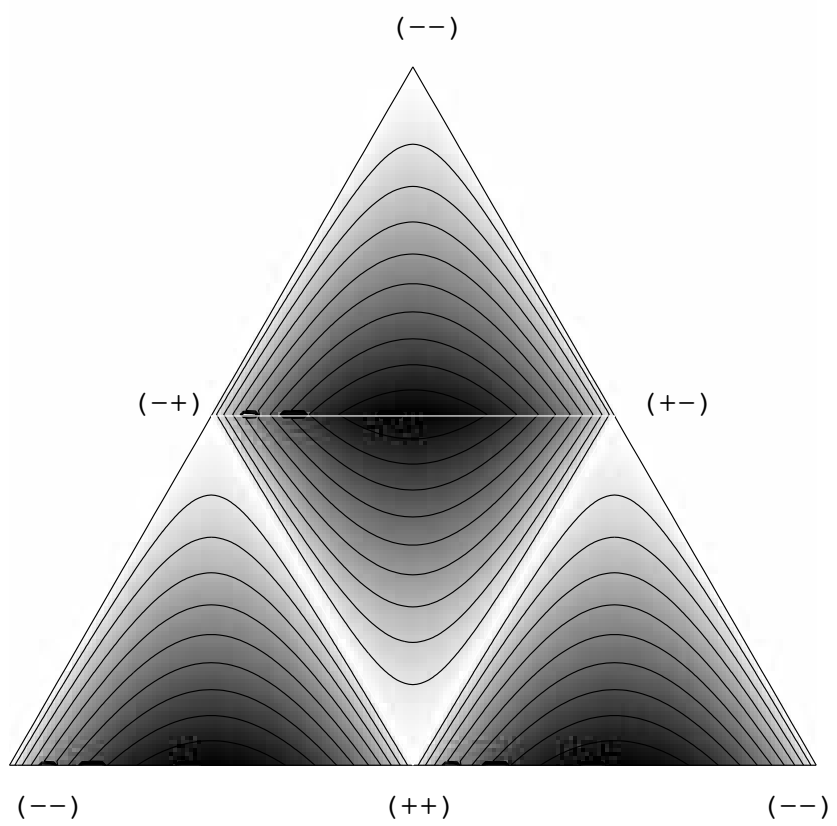

FIG. 4. Entropy of the entanglement, $H_{1}(|\psi\rangle)$ for pure states of the $2 \times 2$ problem at the surface of the $\vartheta$-tetrahedron representing $\mathbb{C} P^{3}$ (dark colors denote large entanglement). Labels refer to the corners representing the standard basis. Copy this picture magnifying, cut out the triangle, fold three times along the edges and glue together into a thetrahedron to enjoy the symmetry of this representation.

\section{Global transformations of mixed states}

Before dealing with the more complicated case of entanglement for the $3 \times 3$ system let us make a detour to have a closer look at the space of mixed quantum states acting in $\mathcal{H}_{N}$. Any state $\rho$ may brought to a diagonal form by a unitary evolution $\rho \rightarrow \rho^{\prime}=U \rho U^{\dagger}$ which preserves the vector of eigenvalues $\vec{d}=\left\{d_{1}, d_{2}, \ldots, d_{N}\right\}$. The normalization condition assures $\operatorname{tr} \rho=\sum_{i=1}^{N} d_{i}=1$.

Any physical operation can be described by a superoperator $\tilde{M}: \rho \rightarrow \rho^{\prime}$ that preserves positivity of the mixed state $\rho$. Strictly speaking the term 'positive' refers to positive semidefinite Hermitian operators, which do not have negative eigenvalues. Moreover, the system may be coupled to an environment, and $\tilde{M}$ may be trivially extended to $\tilde{M} \otimes \mathbb{I}$. A superoperator $\tilde{M}$ is completely positive if for all such extensions $\tilde{M} \otimes \mathbb{I}$ is positive. A completely positive (CP)-map which preserves the trace (normalization of the state is conserved) is called a stochastic map or quantum channel. It is capable to describe any quantum operation including the process of quantum measurement and may be represented in the Krauss form 49.

$$
\rho \rightarrow \rho^{\prime}=\tilde{M}(\rho)=\sum_{i=1}^{k} A_{i} \rho A_{i}^{\dagger},
$$

where the set of $k$ operators $A_{i}$ satisfies the completeness relation, $\sum_{i=1}^{k} A_{i}^{\dagger} A_{i}=\mathbb{I}$.

In the following we shall consider a smaller subset of quantum channels, which may be written in the form $\rho^{\prime}=$ $M(\rho)=\sum_{i=1}^{k} p_{i} U_{i} \rho U_{i}^{\dagger}$, where each operator $U_{i}$ is unitary and the positive coefficients $p_{i}$ sum to unity. These operations, called external random fields [50], are unital, since the maximally mixed state $\rho_{*}=\mathbb{I} / N$ is preserved, $M\left(\rho_{*}\right)=\rho_{*}$. Maps which preserve both the trace and the identity are called bistochastic.

Let $\overrightarrow{d^{\prime}}$ denote the vector of eigenvalues of $\rho^{\prime}=M(\rho)$. Then one can prove 51,52 the following majorization relation, $\overrightarrow{d^{\prime}} \prec \vec{d}$. Using the concept of the $T$-transforms we are going to show the converse: given a vector of eigenvalues $\overrightarrow{d^{\prime}}$ such that $\overrightarrow{d^{\prime}} \prec \vec{d}$ there exists a bistochastic map $M: \rho^{\prime}=M(\rho)$, written $\rho \stackrel{\text { global }}{\longrightarrow} \rho^{\prime}$. Thus both properties are equivalent, 


$$
\rho \stackrel{\text { global }}{\longrightarrow} \rho^{\prime} \Leftrightarrow \overrightarrow{d^{\prime}} \prec \vec{d}
$$

For simplicity we start discussing the $N=3$ case, represented in Fig. 5. Consider a state $\rho$ with spectrum $\vec{d}$, (its components are ordered as $\left.d_{1} \geq d_{2} \geq d_{3}\right)$ and a transformation $M_{12}(\rho)=w_{12} U_{12} \rho U_{12}^{\dagger}+\left(1-w_{12}\right) \rho$. The unitary matrix $U_{12}$ acts as an identity on all but two first components, (labeling the matrix) which get mixed by the orthogonal submatrix: $\left(U_{12}\right)_{11}=\left(U_{12}\right)_{12}=\left(U_{12}\right)_{22}=1 / \sqrt{2}$ and $\left(U_{12}\right)_{21}=-1 / \sqrt{2}$. In this way $M_{1}$ moves $\rho$ along the horizontal line joining $\vec{d}$ with $\vec{d}_{a}=\left(d_{12}, d_{12}, d_{3}\right)$, where $d_{12}=\left(d_{1}+d_{2}\right) / 2$. The length of this move is controlled by the weight parameter $w_{12}$. An analogous transformation $M_{23}(\rho)$ defined by the unitary matrix $U_{23}$ which preserves the first eigenvalue $d_{1}$, allows one to obtain any state along the line between $\vec{d}$ and $\vec{d}_{b}=\left(d_{1}, d_{23}, d_{23}\right)$, where $d_{23}=\left(d_{2}+d_{3}\right) / 2$. It is easy to see that any state with the spectrum fulfilling $\vec{d}^{\prime} \prec \vec{d}$ (shaded region in Fig. 5a), may be reached by a superposition of operations $M_{i j}$, which correspond to $T$-transforms. Observe that the lines limiting the accessible region are parallel to the isoentropy curves $H_{0}$ and $H_{\infty}$, respectively.

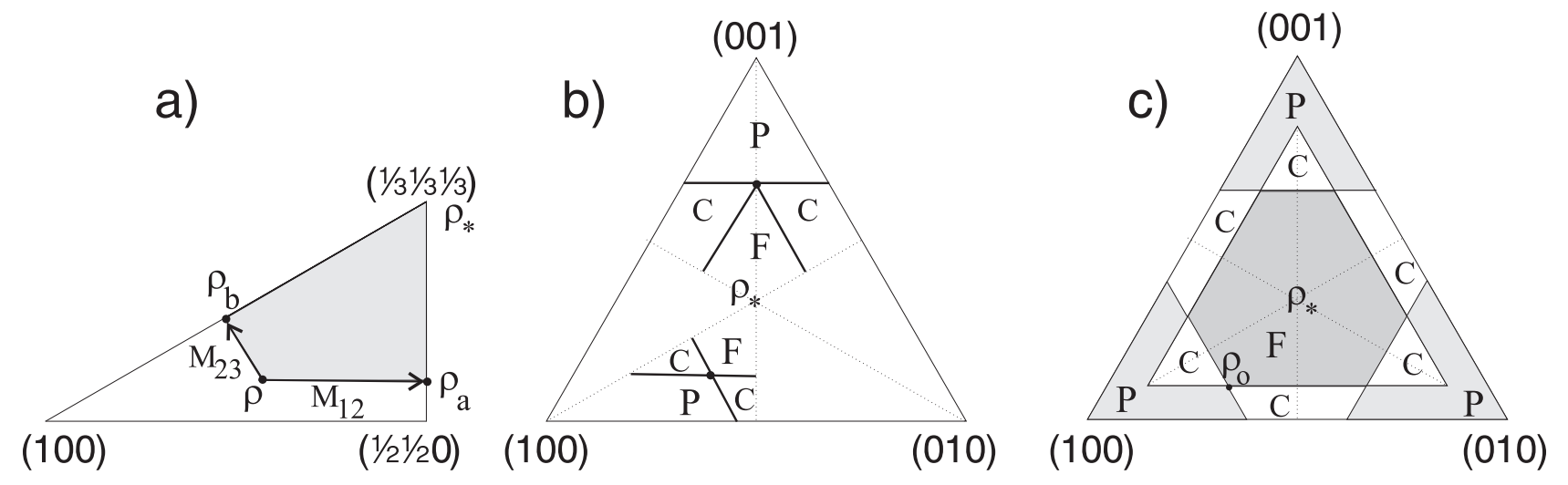

FIG. 5. Simplex of eigenvalues for $N=3$ density matrices: corners represent pure states, center the maximally mixed state $\rho_{*}=\mathbb{I} / 3$; (a) asymmetric part of the simplex (Weyl chamber), for which $d_{1} \geq d_{2} \geq d_{3}$. Evolution governed by two $T$-transforms defines the shaded region accessible from $\rho$; (b) the shape of the 'light cone' depends on the degeneracy of the spectrum: $F$ denotes Future, $P$ Past, and $C$ the non comparable states; (c) splitting of the entire simplex into three parts with permutation operation allowed.

This construction is simply generalized for arbitrary $N$ : the accessible region of states with spectra majorized by $\vec{d}$ is a convex polytope with $2(N-1)$ faces. The point $\vec{d}$ sits in one of its corners, which joins $N-1$ edges - lines generated from $\rho$ by the transformations $M_{i, i+1}$, where $i=1, \ldots, N-1$. Therefore every face forming the part of boundary of the accessible region, (corresponding to the light cone in special relativity), may be generated by a sequence of $(N-2)$ T-transforms acting on $\vec{d}$. The first transform, $M_{1,2}$, moves $\rho$ along a line paralell to the closest face of the simplex, so the entropy $H_{0}$ is conserved. All other transforms, $M_{i, i+1}$, do not influence the largest component, $d_{1}$, so the entropy $H_{\infty}$ remains unchanged.

The majorization property $\overrightarrow{d^{\prime}} \prec \vec{d}$ assures that the Von Neumann entropy and all other entropies $S_{\alpha}$ for $\alpha \geq 0$ grow (strictly speaking do not decrease) during the time evolution governed by random external fields $M$. Note that the factor $1 /(1-\alpha)$ in the definition (2.4) is crucial to assure the Schur convexity of $H_{\alpha}$. For any state $\rho$ it is easy to specify the set of all states $\rho^{\prime}$ accessible from it by external random fields. For these states, denoted in Fig. 5b by $F$ as 'Future', all generalized entropies satisfy $S_{\alpha}\left(\rho^{\prime}\right) \geq S_{\alpha}(\rho)$. The complementary set of all states ' $\rho$ which may be transformed by $M$ into $\rho$ is denoted by $P$ as 'Past'. Obviously $S_{\alpha}(\rho) \geq S_{\alpha}\left({ }^{\prime} \rho\right)$. There exists also a set $C$ of noncomparable states, which are not ordered with respect to $\rho$ by the majorization relation. This structure is thus analogous to the special relativity picture of the light cone. The actual shape of the 'cone' (e.g. the size of the angle 'Future') depends on the degeneracy of $\vec{d}$ as shown in Fig. 5b: in the generic case it is equal to $2 \pi / 3$, while at a bisetrix it shrinks to $\pi / 3$. The 'world line' crossing the point $\rho$ and representing a possible trajectory in the space of density matrices is located inside the accessible region $F$.

The simplex can be divided into $N$ ! asymmetric simplices (called Weyl chambers in group theory), which differ by the permutation of the components of the vector $\vec{d}$. In the discussed case $N=3$ three bisetrices split the simplex into 6 right triangles, one of which is shown in Fig. 5a. A permutation of the components of $\vec{d}$, which might be considered 
as a special unitary case of the random fields $M$, maps $\vec{d}$ into a corresponding symmetric point in one of the other 6 right triangles. Therefore, in the generic case of a non-degenerate spectrum of $\rho$, the entire decomposition of the $N=3$ equilateral triangle of eigenvalues looks as shown in Fig 5c. The set 'Future' has the form of a hexagon, which contains two triples of equal sides, and becomes regular for $\vec{d}=(3,2,1) / 6$, or in general for $\vec{d}=(1 / 3+x, 1 / 3,1 / 3-x)$, where $x \in(0,1 / 3]$. If the spectrum of $\rho$ is degenerate (the vector $\vec{d}$ is located at a bisetrix), the hexagon reduces to an equilateral triangle.

It is not difficult to understand how this construction works in higher dimensions: the set 'Future' is a convex polytope, defined by the convex hull of $N$ ! points obtained from $\vec{d}$ by permutations of its components. This set contains $N$ ! identical simplices surrounding $\rho_{*}$ and is topologically equivalent to a ball. It consists of $N$ ! vertices (one for each permutation), each joining $N-1$ edges. Thus in the generic case this polytope has $N !(N-1) / 2$ edges and $2^{N}-2$ (hyper)faces of dimension $N-2$. For $N=4$ it is a polyhedron with 24 vertices, 36 edges and 14 faces; if the vertex at $\vec{d}$ lies on the line

$$
\vec{d}(x)=\frac{x}{6}(3,2,1,0)+\frac{1-x}{4}(1,1,1,1) ; \quad 0 \leq x \leq 1
$$

then we obtain an Archimedean solid known as the truncated octahedron whose surface is composed of 8 regular hexagons and 6 squares. By definition an Archimedean solid has regular but not equal faces [53]. If the eigenvalues are degenerate, the polytope degenerates as well. It is amusing to notice that a number of Platonic and Archimedean solids appear for special choices of $\vec{d}$. In particular there is a surface in the simplex at which the degeneracy pattern is $\vec{d}=\left(x_{0}, x, x, x_{3}\right)$. If the vertex sits at a particular line on this surface then the polytope is the Archimedean cub-octahedron. Similarly, the surfaces determined by $\vec{d}=\left(x_{0}, x_{1}, x, x\right)$ or $\vec{d}=\left(x, x, x_{2}, x_{3}\right)$ contain lines giving rise to the truncated tetrahedron. On the lines $\vec{d}=\left(x_{0}, x, x, x\right)$ and $\vec{d}=\left(x, x, x, x_{3}\right)$ we obtain regular tetrahedra while the line $\vec{d}=(x, x, y, y)$ yields regular octahedra. So much about the 'Future'. The set 'Past' consists of $N$ disjoint parts occupying neighbourhoods of each corner, while $C$ consists of $2^{N}-2$ parts, each for every face of the set 'Future'. The flat geometry of the simplex of eigenvalues has a concrete meaning, since the Euclidean distance between any two points of a Weyl chamber (Fig. 5a) is equal to the Hilbert-Schmidt distance between the global orbits they generate - see Appendix A.

Note that the analogy with special relativity does not work in the simplest case of $N=2$, for which all measures of the degree of mixing are equivalent. All of them become functions of one single parameter, (say, the distance to the center of the Bloch ball), and generate the same order of the density matrices. The situation changes for $N=3$, for which the spectrum of $\rho$ depends on two parameters, as recently discussed by Jozsa and Schlienz 54. They obtained interesting relations between different entropies $H_{\alpha}$, which may also be proved using the techniques of majorization. which may also be proved using the techniques of majorization. For example, if we move in the triangle of eigenvalues along a circle of a constant $R$ and $\operatorname{tr} \rho^{3}$ grows (the entropy $H_{3}$ decreases), then the von Neumann entropy $H_{1}$ increases. Such relations may be figured out by superimposing together different isoentropy curves shown in Fig. 1.

\section{Local transformations of pure states}

Consider now the set of pure states of an $N \times N$ composite system. We divide it into equivalence classes consisting of states that can be transformed into each other by local unitary transformations; the resulting orbit space is precisely a Weyl chamber in the Schmidt simplex. Let $\Lambda_{\psi}=\left\{\lambda_{1}(\psi), \ldots, \lambda_{N}(\psi)\right\}$ represents the vector of the Schmidt coefficients of a state $|\psi\rangle$ ordered in the descending order. Define a deterministic LOCC transformation as one that takes states to states (as opposed to one that transforms a state to a statistical ensemble of states). Nielsen has shown that a deterministic LOCC transformation of a given state $|\psi\rangle$ into $|\phi\rangle$ is possible if and only if the corresponding vectors of Schmidt coefficients satisfy the majorization relation [7]

$$
|\psi\rangle \stackrel{\text { LOCC }}{\longrightarrow}|\phi\rangle \quad \Leftrightarrow \quad \Lambda_{\psi} \prec \Lambda_{\phi}
$$

Note a similarity between this relation concerning the local transformation of pure states and the condition (3.6) concerning the global bistochastic transformations of mixed states.

Any pure state $|\psi\rangle$ allows one to split the Schmidt simplex, representing local orbits of pure states, into three regions: 'Future' $F$, 'Past' $P$, and non-comparable, $C$. This structure for $N=3$ is shown in Fig. 6 . Although Fig. 5 describes an entirely different physical problem, both picures are almost identical. The only difference consists in the arrow of time: the 'Past' for the evolution in the space of density matrices corresponds to the 'Future' for the 
local entanglement transformations and vice versa. The triangles plotted here should not be confused with Fig. 4. In fact the analogous plot for $N=2$ would contain only one line, say joining the states $(++)$ and $(--)$, along which the entanglement takes all values in $[0, \ln 2]$. On the other hand, the triangle shown in Fig. 6 may be given a direct geometrical interpretation, provided it is deformed into an octant of the sphere. Formally this may be achieved by the transformation $\left\{\lambda_{1}, \ldots, \lambda_{N}\right\} \rightarrow\left\{\lambda_{1}^{2}, \ldots, \lambda_{N}^{2}\right\}$. Then the length of the arc joining two points of the Weyl chamber (which covers $1 / 48$ of the sphere) is proportional to the Fubini-Study distance between the local orbits they generate - see Appendix A.

In general, all different measures of mixing increase during the operations considered and all the measures of entanglement decrease. In particular it follows that all generalized entropies $H_{\alpha}(\Lambda)$ and their convex functions belong to the class of entanglement monotones which do not increase during local operations [8]. For example $H_{\infty}(\Lambda)$ equals minus maximal Schmidt coefficient, $-\lambda_{1}$, a simple entanglement monotone discussed by Vidal [8]. The entropies $H_{\alpha}$ satisfy thus the conditions (E1)-(E3) required for a measure of the entanglement. The maximal fidelity of a pure state, $F_{\max }(\psi)=\left(\sum_{i=1}^{N} \sqrt{\lambda_{i}}\right)^{2} / N$ [63], a convex function of the generalized entropy, $F_{\max }=\exp \left(H_{1 / 2}\right) / N$, is also an entanglement monotone due to the Schur convexity. So is another measure of entanglement called robustness, which is defined as the minimal amount of separable noise that has to be mixed with the analyzed state to wash out completely its quantum correlations [56]8]. For pure states the robustness equals $N F_{\max }-1=\exp \left(H_{1 / 2}\right)-1$.

Another quantity used to characterize the entanglement is called negativity [57,58]. It is based on the concept of partial transposition (transposition in one of the two subsystems). It is known that for any separable state $\rho$ its partial transpose $\rho^{T_{2}}$ is positive [1,2], and the negativity is expressed by the trace norm, $t:=\left|\rho^{T_{2}}\right|_{\operatorname{tr}}-1$, i.e. by the sum of absolute values of the eigenvalues. Negativity is an entanglement monotone and satisfies the axioms (E1)-(E3) [59]. For pure states of $2 \times 2$ system it is equal to concurrence, used by Wootters to calculate the entanglement of formation [42,13]. For any $N \times N$ pure state written in the Schmidt form (2.2), the partially transposed matrix $\rho^{T_{2}}$ has a simple block structure: it consists of $N$ Schmidt numbers at the diagonal (which sum to unity) and $N(N-1) / 2$ blocks of size 2, one for each pair of different indices $(i, j)$. Eigenvalues of each block are $\pm \sqrt{\lambda_{i} \lambda_{j}}$, so the negativity reads

$$
t(|\psi\rangle)=2 \sum_{i>j=1}^{N} \sqrt{\lambda_{i} \lambda_{j}}=\left(\sum_{i=1}^{N} \sqrt{\lambda_{i}}\right)^{2}-1,
$$

which varies from 0 for separable states to $N-1$ for maximally entangled states. It is easy to see that for pure states the negativity is equal to robustness, so is also a function of the quantum Rényi entropy entropy of order one half, $t=\exp \left(H_{1 / 2}\right)-1$. Interestingly, the same behaviour for the pure states is characteristic to the cross norm another measure of entanglement introduced by Rudolph 14. Although negativity $t$ does not satisfy (E4) its nonlinear function, $H_{1 / 2}=\ln (t+1)$, is additive in the general case of arbitrary mixed states, as all other Rényi entropies. On the other hand, the negativity suffers a serious drawback: it is not capable to detect so-called bound entangled states with positive partial transpose, which are known to exist for quantum systems with $\operatorname{dim} \mathcal{H} \geq 8$ 60, 61, .

Let us emphasize that the analogy to special relativity holds for the description of local transformations of pure states entanglement. This analogy was put forward explicitly in the paper by Hwang et al. [27] and used (correctly) to describe a related problem of the mixed states entanglement. As we point out in this work, the same analogy is useful also in investigating the pure states entanglement. However, the authors of [27 discussed only the simplest $2 \times 2$ problem, for which all entanglement measures for pure states generate the same ordering. In the general case this is no longer true. For example the maximal fidelity $F_{\max }$ and the entropy of entanglement $H_{1}$ generate different ordering of pure states for $N>2$, as can be deduced in Fig. 1 for $N=3$. The fact that various entanglement measures generate different ordering of the set of pure states (condition (3.4) is violated), has been raised in [26, 12]. Vidal concludes that it is necessary to use $N-1$ different measures of entanglement to provide a detailed description of the set of pure states of $N \times N$ system. This is a consequence of the fact that there exists exactly $N-1$ independent Schmidt coefficients in the decomposition (2.2). A possible set of entanglement monotones is given by the sum of the $N-k+1$ smallest Schmidt coefficients, $E_{k}=\sum_{i=k}^{N} \lambda_{i}$, with $k=2, \ldots N$ [8]. Note that the sum of $N-1$ smallest coefficients is related to the already mentioned monotone, the largest coefficient, $\lambda_{1}=1-E_{2}$. 


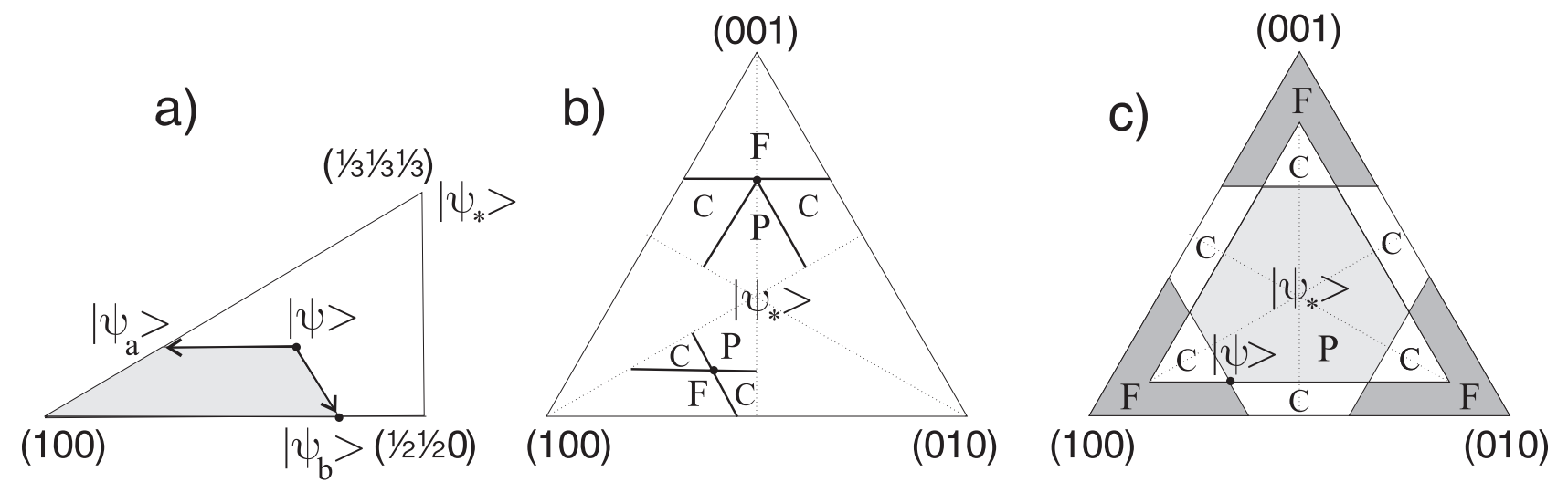

FIG. 6. Simplex of Schmidt coefficients for $3 \times 3$ pure states: corners represent separable states, center the maximally entangled state $\left|\psi_{*}\right\rangle$. Panels (a-c) analogous to these in Fig. 5, observe the opposite direction of the arrow of time. As in Fig. 5 the bounding lines are the level curves of $H_{0}$ and $H_{\infty}$ familiar from Fig. 1.

Pure states with the same set of the Schmidt coefficients (e.g. 6 points in fig. 6c generated be reflections with respect to all bisetrices) are called interconvertible [62], since they (and only they) might be transformed reversibly by local transformations. The generic orbit of pure states equivalent by local unitary operations has $N^{2}-N-1$ dimensions, and this number decreases in the case of a degeneracy in the vector of the Schmidt coefficients [44]. On the other hand, the 'noncomparable' states cannot be joined by a local transformation in any direction. The existence of such pairs of states (analogous to the space-like events in special relativity) was recently discussed in [26,76.62, 63]. In fact, Nielsen conjectured [7] that the probability of picking at random two incomparable states out of the set of all $N \times N$ pure states (according to the natural, rotationally invariant measure) tends to unity in the limit $N \rightarrow \infty$. We may now support this conjecture with a simple geometrical argument: the states close to the faces of the Schmidt simplex are incomparable, (see Fig. 6c) and it is well known that for large dimensional simplices almost all its volume is contained in a thin 'skin' close to its faces. Furthermore, the natural, unitarily invariant measure on $\mathbb{C} P^{N-1}$ generates the Hilbert-Schmidt measure in the Schmidt simplex 47,48, according to which the degeneracies in $\Lambda$ are avoided: the probability distribution exhibits maxima in vicinity of the faces of the simplex, while its minima occur at the center of the simplex and along the symmetry planes (bisetrices of the triangle) including the corners.

\section{E. Probabilistic transformations of pure states}

Finally we discuss the case when the majorization condition (3.8) is not fulfilled, so that deterministic local transformation of the pure state $|\psi\rangle$ into $|\phi\rangle$ is not possible. It is not possible to create entanglement by means of LOCC if the initial state is separable, but it can be shown that if the numbers of nonzero components in both Schmidt vectors are the same then one may still transform $|\psi\rangle$ into $|\phi\rangle$ with a non-zero probability $P$ of success. More precisely it was shown by Vidal [26] that the optimal protocol yields

$$
P(|\psi\rangle \rightarrow|\phi\rangle)=\min _{k \in[1, N]} \frac{\sum_{i=k}^{N} \lambda_{i}(\psi)}{\sum_{i=k}^{N} \lambda_{i}(\phi)}=\min _{k \in[1, N]} \frac{E_{k}(\psi)}{E_{k}(\phi)} .
$$

This statement may be rephrased by saing that the optimal probability $P(|\psi\rangle \rightarrow|\phi\rangle)$ is the greatest weight $p$ such that the submajorization relation holds [63],

$$
\Lambda_{\psi} \prec w p \Lambda_{\phi}
$$

Probabilistic transformations are important because they make entanglement purification procedures possible.

Figure 7 shows the probability of accessing different regions of the Schmidt simplex for pure states of a $3 \times 3$ system for four different initial states $|\psi\rangle$. The shape of the black figure ( $p=1$ represents deterministic transformations) is identical with the set 'Future' in Fig.6. The more entangled final state $|\phi\rangle$ (closer to the maximally entangled state in the center of the triangle), the smaller probability $p$ of a successful transformation. Observe that the contour lines (plotted at $p=0.2,0.4,0.6$ and 0.8 ) are constructed from the iso-entropy lines $H_{\alpha}$ for $\alpha \rightarrow 0$ and $\alpha \rightarrow \infty$ (see Fig. 1.) 

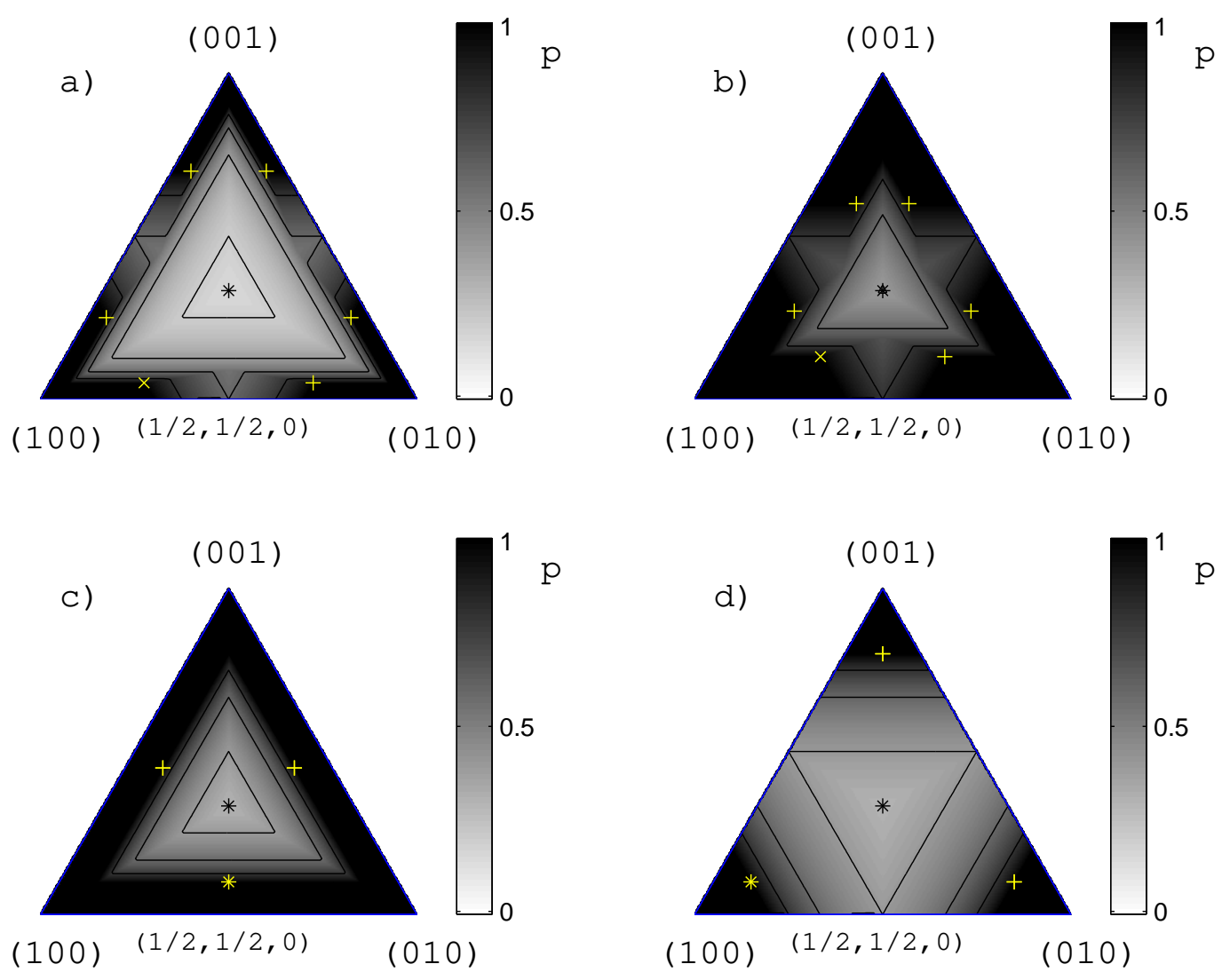

FIG. 7. Probabilistic transformation of pure states in the simplex of Schmidt coefficients for $3 \times 3$ pure states: white $(\times)$ represents the initial state $|\psi\rangle(+)$ the interconvertible states, and black $(*)$ the maximally entangled state. The gray scale represents the probability $p$, with which a given state may be obtained by local transformations from $|\psi\rangle$. Initial state $|\psi\rangle$ is characterized by the Schmidt coefficients $\Lambda=\left(\lambda_{1}, \lambda_{2}, 1-\lambda_{1}-\lambda_{2}\right)$, where $\left(\lambda_{1}, \lambda_{2}\right)=(\mathrm{a})(0.7,0.25),(\mathrm{b})(0.6,0.27),(\mathrm{c})$ $(0.45,0.45)$, and (d) $(0.8,0.1)$. Due to the degeneracy in $\Lambda$ in the last two cases there exist only three different interconverible states.

\section{CONCLUDING REMARKS}

We presented a brief review of various measures of entanglement and ways to define them. Analyzing geometrical properties of the pure states entanglement we have shown that there exist several non-equivalent measures of entanglement which generate different ordering of states. Four different measures often discussed in the literature: robustness of entanglement (negativity or cross norm), entropy of entanglement, Bures distance to the closest mixed state and the distance to the closest pure state are functions of the generalized Rényi-like entropies of the vector of the Schmidt coefficients (2.4) with $\alpha=1 / 2,1,2$ and $\alpha \rightarrow \infty$, respectively, - see Table 1. 


\begin{tabular}{|c|c|c|}
\hline $\begin{array}{l}\text { Measure of } \\
\text { entanglement }\end{array}$ & $\begin{array}{l}\begin{array}{l}\text { Formula for } \\
\text { pure states }\end{array} \\
\end{array}$ & $\begin{array}{l}\text { Monotone function } \\
\text { of } H_{\alpha} \text { with }\end{array}$ \\
\hline$\overline{\text { Schmidt rank }}$ & number of nonzero $\lambda_{i}$ & $\alpha=0$ \\
\hline Maximal fidelity & 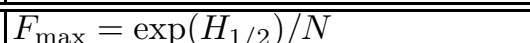 & \multirow{4}{*}{$\alpha=\frac{1}{2}$} \\
\hline Negativity & $t=\exp \left(H_{1 / 2}\right)-1$ & \\
\hline Robustness & $N F_{\max }-1=\exp \left(H_{1 / 2}\right)-1=t$ & \\
\hline Cross-norm & & \\
\hline$\overline{\text { Entropy of entanglement }}$ & $E=H_{N}=-\sum_{i=1}^{N} \lambda_{i} \ln \lambda_{i}$ & \multirow{4}{*}{$\alpha=1$} \\
\hline Relative entropy & $\min _{S E P} S\left(\rho \| \rho_{s}\right)=E$ & \\
\hline Entropy of formation & $E_{F}=E$ & \\
\hline Entropy of distillation & $E_{D}=E$ & \\
\hline \begin{tabular}{|l|} 
Minimal Bures distance \\
to a separable (mixed) state \\
\end{tabular} & $\min _{S E P}\left(D_{B}\right)=2\left[1-\exp \left(-\frac{1}{2} H_{2}\right)\right]$ & \multirow[t]{2}{*}{$\alpha=2$} \\
\hline Generalized concurrence & & \\
\hline $\begin{array}{l}\text { Minimal distance to } \\
\text { a separable (pure) state }\end{array}$ & & \multirow{5}{*}{$\alpha \rightarrow \infty$} \\
\hline Fubini-Study distance & $\min _{S E P}\left(D_{F S}\right)=\arccos \left[\sqrt{\lambda_{\max }}\right]$ & \\
\hline trace distance & $\min _{S E P}\left(D_{t r}\right)=2 \sqrt{1-\lambda_{\max }}$ & \\
\hline Hilbert-Schmidt distance & $\min _{S E P}\left(D_{H S}\right)=\sqrt{2\left(1-\lambda_{\max }\right)}$ & \\
\hline Bures distance & $\min _{S E P}\left(D_{B}\right)=\sqrt{2\left(1-\sqrt{\lambda_{\max }}\right)}$ & \\
\hline
\end{tabular}

Table. 1. Measures of the entanglement for $N \times N$ bi-partite system ordered according to the properties at the manifold of pure states.

Out of these measures only the entropy of entanglement is additive, if pure states are concerned. The remaining quantities (not equivalent to $S$ ) are not, but each measure may be related with a certain additive generalized entropy $H_{\alpha}$. Thus no single measure seems to be a priori distinguished. On the other hand, some of them may be preferred by certain experimentally feasible protocols of local manipulations of the pure states, (e.g. the distillation of entanglement), or by the property (E5') of the asymptotic continuity [8,9].

The above analysis has direct consequences for the more complicated problem of the entanglement of mixed states. Consider the definition of entanglement of formation of a mixed state [6]

$$
\operatorname{EoF}(\rho):=\min \sum_{i=1}^{L} p_{i} E\left(\left|\psi_{i}\right\rangle\right), \quad p_{i}>0,
$$

where the minimum is taken with over all pure states decompositions $\rho=\sum_{i=1}^{L} p_{i}|\psi\rangle\langle\psi|$ of a finite length $L$, normalized by $\sum_{i=1}^{L} p_{i}=1$. In this expression one makes use of the entropy of entanglement, $E(|\psi\rangle)=H_{N}(|\psi\rangle)$, but one may also apply other generalized entropies $H_{\alpha}(|\psi\rangle)$ to generate reasonable measures of the mixed states entanglement [8]. It is easy to see that these generalized entropies of formation induce different orderings in the set of density matrices.

Time evolution of pure states under such transformations reveals an analogy to special relativity: We first divide the set of pure states into equivalence classes consisting of the orbits under local unitary transformations. Then we find that for each point in this space of equivalence classes of pure states there exists a 'light cone' which divides the space into the 'Future' (F), the 'Past' (P) and the non-comparable states (C). In the Schmidt simplex the role of the light cone is played by the accessible region limited by (hyper)faces spanned by any set of $(N-2)$ edges determined by the $T$-transforms. Thus it is a generalized cone bounded by hyperplanes. This "causal structure" (to pursue the analogy to relativity) induces a partial ordering of the states but there is no complete ordering and in this sense then there does not exist a preferred measure of entanglement, just as there does not exist an absolute time in relativity. On the other hand (unlike in special relativity) the underlying "spacetime" is not maximally symmetric, although it comes close to be so. In such situations one often finds that one time slicing is more "natural" than another. Pursuing the analogy further one expects that a particular measure of entanglement can be singled out by the physical context.

It is a pleasure to thank Johan Brännlund, Paweł Horodecki, Marek Kuś and Thomas Wellens for inspiring discussions. We appreciate helpful remarks raised by the referee. Financial support by Komitet Badań Naukowych in Warsaw under the grant 2P03B-072 19, the NFR, and the European Science Foundation are gratefully acknowledged. 


\section{APPENDIX A: DISTANCES IN WEYL CHAMBERS AS DISTANCES BETWEEN ORBITS}

Before discussing possible interpretation of the distance in Weyl chamber let us formulate two simple lemmas related to the majorization theory.

Lemma 1. Let $\vec{x}, \vec{y}$ and $\vec{z}$ be three normalized probability vectors with $N$ components in the descending order each, and let $\vec{z} \prec \vec{y}$. Then the scalar products fulfill

$$
\vec{x} \cdot \vec{z} \leq \vec{x} \cdot \vec{y}
$$

Proof: For simplicity we start with $N=3$ and consider the difference $\vec{x} \cdot \vec{y}-\vec{x} \cdot \vec{z}$. It is equal to the sum of three terms, $x_{1}\left(y_{1}-z_{1}\right)+x_{2}\left(y_{2}-z_{2}\right)+x_{3}\left(y_{3}-z_{3}\right)=\left(x_{1}-x_{2}\right)\left(y_{1}-z_{1}\right)+\left(x_{2}-x_{3}\right)\left(y_{1}+y_{2}-z_{1}-z_{2}\right)+x_{3}\left(y_{1}+y_{2}+y_{3}-z_{1}-z_{2}-z_{3}\right)$, the first two are non-negative and the last one vanishes. In the similar way, for an arbitrary $N$ we represent $\sum_{k=1}^{N} x_{k}\left(y_{k}-z_{k}\right)$

as $\sum_{k=1}^{N-1}\left(x_{k}-x_{k+1}\right) \sum_{i=1}^{k}\left(y_{i}-z_{i}\right)$. Due to ordering of the components of $\vec{x}$ and the majorization relation $\vec{z} \prec \vec{y}$ this expression contains non-negative terms only, and this proves the thesis (A1) $\square$.

Although the next lemma we shall use is closely related with recent results of Nielsen [38], we formulate it in the form most suitable for our purposes and for consistency provide its proof.

Lemma 2. Let $\rho$ be a density matrix, $\vec{\lambda}$ its spectrum and $\vec{x}$ denotes the diagonal elements of $\rho$ represented in a certain basis. Then $\vec{x} \prec \vec{\lambda}$.

Proof: Any density matrix may be diagonalized, $\rho=U \lambda U^{\dagger}$. Thus the diagonal elements read $x_{i}=\rho_{i i}=U_{i j} \lambda_{j} U_{j i}^{\dagger}$. Introducing a unistochastic matrix $D_{i j}:=\left|U_{i j}\right|^{2}$ we may then write $\vec{x}=D \vec{\lambda}$, so due to the Horn lemma [36] the majorization relation $\vec{x} \prec \vec{\lambda}$ holds $\square$.

Now we are ready to formulate statements concerning the Weyl chamber which contains the set of diagonal density matrices with all entries ordered.

Proposition 1: Let $h$ and $g$ be two diagonal density matrices of size $N$ with all components in a descending order, so they belong to the same Weyl chamber. Let $U$ and $V$ denote arbitary unitary matrices of size $N$. Then

a) $D_{H S}(h, g) \leq D_{H S}\left(U h U^{\dagger}, V g V^{\dagger}\right)$,

b) the Euclidean distance between any two points in the Weyl chamber is equal to the Hilbert-Schmidt distance between the global orbits they generate.

Proof:. Let $W=U^{\dagger} V$. Applying the definition $\left[D_{H S}(h, g)\right]^{2}=\operatorname{Tr}(h-g)^{2}$ we may rewrite the difference of the squared distances, $Q=\left[D_{H S}\left(U h U^{\dagger}, V g V^{\dagger}\right)\right]^{2}-\left[D_{H S}(h, g)\right]^{2}$ as $-2 \operatorname{Tr} W^{\dagger} h W g+2 \operatorname{Tr} h g$. Let $\vec{h}^{\prime}$ denote the vector of the diagonal elements of $W^{\dagger} h W$, so the difference we compute is $Q=2\left(\vec{h} \cdot \vec{g}-\vec{h}^{\prime} \cdot \vec{g}\right)$. Applying lemma 2 we obtain $\vec{h}^{\prime} \prec \vec{d}$, and using lemma 1 we conclude that the difference $Q$ is non-negative, which gives the statement a). Thus the HS-distance between two orbits, $U h U^{\dagger}$ and $V g V^{\dagger}$, equal to the HS-distance between closest points $h$ and $g$, reads $D_{H S}(h, g)=\left[\sum_{i=1}^{N}\left(h_{i}-g_{i}\right)\right]^{1 / 2}$. This is just the standard expression for the Euclidean distance between two points in the Weyl chamber which represent both diagonal density matrices, $h$ and $g \square$.

A similar statement may also be formulated for other distances (trace, Bures), but they do not allow for such a simple geometric interpretation as does the Hilbert-Schmidt distance used above. Note that the assumption that both density matrices belong to the same Weyl chamber is crucial, since a generic orbit visits the simplex of eigenvalues $N$ ! times, corresponding to permutations of the components in the spectrum.

The above proposition clarifies the geometry of Fig. 5a, which shows the asymmetric part of the simplex of eigenvalues of the density matrices. To characterize in an analogous way the part of the Schmidt simplex of pure states presented in Fig. 6a, we need to change its geometry. The equilateral triangle from Fig. $6 \mathrm{~b}$ has to be deformed into an octant of the sphere. More precisely, we take the vector of the Schmidt coefficients $\vec{\lambda}=\left\{\lambda_{1}, \ldots, \lambda_{N}\right\}$ of a pure state of a $N \times N$ bi-partite system and transform it to $\left\{\lambda_{1}^{2}, \ldots, \lambda_{N}^{2}\right\}$. Then the $N-1$ dimensional Schmidt simplex is mapped into a $1 / 2^{N+1}$ part of the hypersphere $S^{N-1}$, equipped with a natural Riemannian distance (for $N=3$ it is just the length of the arc at the sphere $S^{2}$ ). This geometry allows us to formulate an analogous

Proposition 2: Let $\lambda$ and $\mu$ be two vectors of Schmidt coefficients, representing two pure states $|\phi\rangle$ and $|\psi\rangle$ of a $N \times N$ bi-partite system. Assume that all their components are ordered in a descending manner, so both points belong to the same Weyl chamber. Let $U=U_{1} \otimes U_{2}$ and $V=V_{1} \otimes V_{2}$ denote arbitary local unitary transformations. Then

a) $D_{F S}(|\psi\rangle,|\phi\rangle) \leq D_{F S}\left(U_{1} \otimes U_{2}|\psi\rangle, V_{1} \otimes V_{2}|\phi\rangle\right)$,

b) the Riemannian distance between any two points in the Weyl chamber (a subset of the deformed Schmidt simplex) is equal to the Fubini-Study distance between the local orbits they generate.

Proof:. In the analogy to the proposition 1, the proof of this proposition may be based on the lemmas 1 and 2 . Alternatively, one can make use of lemma 1 of a recent paper of Vidal et al. [63], which states that the maximal 
fidelity $F=\left|\left\langle\phi\left|U_{1} \otimes U_{2}\right| \psi\right\rangle\right|^{2}$ is obtained for $U_{1}=U_{2}=\mathbb{I}$ and is equal to $F_{\max }=\left(\sum_{k=1}^{N} \sqrt{\lambda_{k} \mu_{k}}\right)^{2}$. This fact allows us to establish part b) of the proposition, since the Riemannian distance between two points in the Weyl chamber $D_{R}(\vec{\lambda}, \vec{\mu})$ is equal to $\cos ^{-1}\left(\sqrt{F_{\max }}\right)=\frac{1}{2} \cos ^{-1}\left(2 F_{\max }-1\right)$ and coincides with the Fubini-Study distance between pure states $|\phi\rangle$ and $|\psi\rangle \square$.

Note that the both propositions resemble the situation for a fibre bundle, where a metric on the total bundle space gives a shortest distance between the fibres that can then be used to define a metric on the base manifold. However, in our situation not all orbits need to be the same: the dimensionality of local orbits generated by degenerated Schmidt vectors (located at the boundary of the Weyl chamber) is analyzed in ref [44].

[1] A. Peres, Phys. Rev. Lett. 77, 1413 (1996).

[2] R. Horodecki, P. Horodecki and M. Horodecki, Phys. Lett. A 223, 1 (1996)

[3] M. Lewenstein, D. Bruss, J. I. Cirac, B. Kraus, M. Kuś, J. Samsonowicz, A. Sanpera, and R. Tarrach, J. Mod. Opt. 47, 2481 (2000).

[4] M. Horodecki, P. Horodecki and R. Horodecki, in Quantum information - an introduction to basic theoretical concepts and experiments, Springer, Berlin 2001.

[5] C. H. Bennett, H. J. Bernstein, S. Popescu and B. Schumacher, Phys. Rev. A53 2046 (1996).

[6] C. H. Bennet, D.P. DiVincenzo, J.A. Smolin and W.K. Wootters, Phys. Rev. A 54, 3824 (1996).

[7] M. A. Nielsen, Phys. Rev. Lett. 83436 (1999)

[8] G. Vidal, J. Mod. Opt. 47, 355 (2000).

[9] M.J. Donald, M. Horodecki and O. Rudolph, arXiv preprint quant-ph/0105017.

[10] V. Vedral and M. B. Plenio, Phys. Rev. A 57, 1619 (1998).

[11] M. Horodecki, P. Horodecki and R. Horodecki Phys. Rev. Lett. 84, 2014 (2000).

[12] S. Virmani and M.B. Plenio, Phys. Lett. A 268, 31 (2000).

[13] W. K. Wootters, Quant. Inf. Comp. 1, 27 (2001).

[14] O. Rudolph, arXiv preprint quant-ph/0005011 (2000).

[15] M. Horodecki, Quant. Inf. Comp. 1, 3 (2001).

[16] A. Peres, Quantum Theory: Concepts and Methods, Kluwer, Dordrecht 1993.

[17] N. Linden, S. Popescu and A. Sudbery, Phys. Rev. Lett. 83, 243 (1999).

[18] M. Grassl, M. Rötteler, and T. Beth, Phys. Rev. A 58, 1833 (1998).

[19] B.-G. Englert and N. Metwally, J. Mod. Opt. 472221 (2000)

[20] Y. Makhlin, arXiv preprint quant-ph/0002045.

[21] H. A. Carteret and A. Sudbery, J. Phys. A 33, 4981 (2000).

[22] S. J. Lomonaco, arXiv preprint quant-ph/0101120

[23] M. Kuś and K. Życzkowski, Phys. Rev. A 63, 032307-13 (2001)

[24] C. Witte, M. Trucks, Phys. Lett. A257, 14 (1999).

[25] M. Lewenstein and A. Sanpera Phys. Rev. Lett. 80, 2261 (1998).

[26] G. Vidal, Phys. Rev. Lett. 83, 1046 (1999).

[27] W.Y. Hwang, D. Ahn, S.W. Hwang and J. Lee, arXiv preprint quant-ph/0011010

[28] E. Schmidt, Math. Annalen 63, 433 (1906). Kluver, Dordrecht 1993.

[29] A. Ekert and P. L. Knight, Am. J. Phys. 63, 415 (1995).

[30] O. Rudolph, arXiv preprint quant-ph/0105104 (2001).

[31] A. Rényi, in. Proc. Fourth. Berkeley Symp. Math. Stat. Prob. 1960, Vol. I, p.547, Berkeley, 1961.

[32] J. N. Kapur, Measures of Information and Their Applications, John Wiley \& Sons, New York 1994.

[33] A. Wehrl, Rev. Mod. Phys. 50, 221 (1978).

[34] R. Horodecki, P. Horodecki and M. Horodecki, Phys. Lett. A 210, 377 (1996)

[35] A. W. Marshall and I. Olkin, Inequalities: Theory of Majorization and its Applications, Academic Press, New York 1979.

[36] A. Horn, Am. J. Math. 76, 620 (1954).

[37] R. Bhatia, Matrix Analysis, Springer-Verlag, New York 1997.

[38] M. A. Nielsen, Phys. Rev. A62, 052308 (2001)

[39] M. A. Nielsen and J. Kempe, Phys. Rev. Lett. 86, 5184 (2001).

[40] D. J. C. Bures, Trans. Am. Math. Soc. 135, 199 (1969).

[41] P. Rungta, V. Buzek, C. M. Caves, M. Hillery and G.J. Millburn, arXiv preprint quant-ph/0102040.

[42] W.K. Wootters, Phys. Rev. Lett. 80, 2245 (1998).

[43] R. B. Lockhart and M. J. Steiner arXiv preprint quant-ph/0009090 
[44] M. Sinołęcka et al. to be published.

[45] Arvind, K. S. Mallesh and N. Mukunda, J. Phys. A 30, 2417 (1997).

[46] J. Eisert and M. B. Plenio, J. Mod. Opt. 46, 145 (1999).

[47] M.J.W. Hall Phys. Lett. A 242, 123 (1998).

[48] K. Życzkowski and H.-J. Sommers, arXiv preprint quant-ph/0012101; J. Phys. A, (2001) in press

[49] K. Krauss, Ann. Phys., 64, 311 (1971),

[50] R. Alicki and K. Lendi, Quantum Dynamical Semigroups and Applications, Lecture Notes in Physics vol. 286, Springer, Berlin 1987.

[51] A. Uhlmann, Wiss. Z. Karl-Marx-Univ. Leipzig 20, 633 (1971).

[52] M. A. Nielsen, Phys. Rev. A 63, 022114 (2001)

[53] P.R. Cromwell, Polyhedra, Cambridge University Press, Cambridge 1997.

[54] R. Jozsa and J. Schlienz, Phys. Rev. A 62, 012301, (2000).

[55] G. Vidal, D. Jonathan and M. A. Nielsen, Phys. Rev. A 62, 012304 (2000).

[56] G. Vidal and R. Tarrach, Phys. Rev. A 59, 141 (1999).

[57] K. Życzkowski, P. Horodecki, A. Sanpera and M. Lewenstein Phys. Rev. A 58, 883, (1998).

[58] K. Życzkowski, Phys. Rev. A 60, 3496 (1999).

[59] G. Vidal and R. F. Werner, arXiv preprint quant-ph/0102117

[60] P. Horodecki, Phys. Lett. A 232, 333 (1997).

[61] M. Horodecki, R. Horodecki, and P. Horodecki, Phys. Rev. Lett. 80, 5239 (1998).

[62] D. Jonathan and M. B. Plenio, Phys. Rev. Lett. 83, 3566 (1999).

[63] G. Vidal, D. Jonathan and M. A. Nielsen, Phys. Rev. A 62, 012304 (2000). 